OPEN ACCESS

Edited by:

Mauro D'Onofrio,

University of Padua, Italy

Reviewed by:

Paola Severgnini,

INAF-Osservatorio Astronomico di

Brera, Italy

Anna Lia Longinotti, National Institute of Astrophysics,

Optics and Electronics, Mexico

${ }^{*}$ Correspondence:

Francoise Combes

francoise.combes@obspm.fr

Specialty section:

This article was submitted to Milky Way and Galaxies,

a section of the journal

Frontiers in Astronomy and Space

Sciences

Received: 30 July 2017

Accepted: 16 August 2017

Published: 21 September 2017

Citation:

Combes F (2017) AGN Feedback and

Its Quenching Efficiency.

Front. Astron. Space Sci. 4:10.

doi: 10.3389/fspas.2017.00010

\section{AGN Feedback and Its Quenching Efficiency}

\author{
Francoise Combes* \\ Observatoire de Paris, LERMA, Centre National de la Recherche Scientifique, College de France, PSL, Sorbonne University \\ UPMC, Paris, France
}

In the last decade, observations have accumulated on gas outflows in galaxies, and in particular massive molecular ones. The mass outflow rate is estimated between 1 and 5 times the star formation rate. For the highest maximal velocities, they are driven by AGN; these outflows are therefore a clear way to moderate or suppress star formation. Some of the most convincing examples at low redshift come from the radio mode, when the radio jets are inclined toward the galaxy plane, or expand in the hot intra-cluster medium, in cool core clusters. However, AGN feedback can also be positive in many occasions, and the net effect is difficult to evaluate. The quenching efficiency is discussed in view of recent observations.

Keywords: galaxies, active galaxy nuclei, black holes, outflows, molecules

\section{TYPES OF FEEDBACK}

Cosmological simulations in the CDM scenario predict too many galaxies at both ends of the mass function. If it is possible to suppress star formation through supernovae feedback in dwarf galaxies, we have to rely on AGN feedback to quench star formation in massive galaxies. There are two main modes of AGN feedback: first, the quasar mode, called also radiative mode or wind mode. This occurs when the AGN luminosity is high, close to Eddington, mainly for young QSO at high redshift. Due to radiation pressure on the ionized gas, the nucleus reaches its gas accretion limit, and begins to eject some gas in a wind. Since the Eddington luminosity $L_{E d d}$ is proportional to $M_{B H} / \sigma_{T}$, where $M_{B H}$ is the supermassive black hole mass and $\sigma_{T}$ the Thomson cross section, the Eddington limitation in BH growth might explain the M- $\sigma$ relation, i.e., $M_{B H} \propto f \sigma_{T} \sigma^{4}$, where $f$ is the gas fraction, and $\sigma$ the central velocity dispersion. The same consideration can be made, when a central starburst reaches its Eddington luminosity, with radiation pressure on dust. Now the cross section is $\sigma_{d}$, which is 1,000 times higher than $\sigma_{T}$. This could lead to a limitation of the bulge mass to $1,000 M_{B H}$, quite close to the observed $M_{\text {bulge }} / M_{B H}$ ratio (Fabian, 2012).

The second feedback scenario is the radio mode, or kinetic mode, due to radio jets. This takes place in very low luminosity AGN, when $\mathrm{L}<0.01 L_{E d d}$, mainly at low redshift. It is frequent in relatively massive galaxies, like the radio-loud ellipticals, powered by a radiatively inefficient flow (ADAF). A particular example of this feedback mode is the moderation of cooling flows in clusters, through radio-jets from the brightest central galaxy (BCG). It is observed also in low-luminosity AGN, like Seyfert galaxies (Combes et al., 2013; Dasyra et al., 2016).

Frequently, star formation and nuclear activity are associated, and it is difficult to disentangle the supernovae and AGN feedback. Galactic winds coming from a starburst (like the prototypical M82) are in general less violent, with smaller maximum velocity, and un-collimated. An example of both is provided by the galaxy merger NGC 3256, an ultra-luminous starburst at $z=0.01$. ALMA 
observations of the molecular gas (through the $\mathrm{CO}(3-2)$ line) have revealed high-velocity wings in both nuclei, the face-on N3256N and almost edge-on N3256S (Sakamoto et al., 2014). For the latter, the outflow is highly collimated, and likely due to an AGN (cf. Figure 1). The derived maximum velocity is $\sim 2,000 \mathrm{~km} / \mathrm{s}$ out to $300 \mathrm{pc}$, and corresponds to $50 \mathrm{M}_{\odot} / \mathrm{yr}$. For the northern galaxy, the maximum velocity is $\sim 750 \mathrm{~km} / \mathrm{s}$, and the outflow rate of $60 \mathrm{M}_{\odot} / \mathrm{yr}$. In both cases, these rates are comparable to the star formation rate, showing that the implied quenching is significant. The time-scale to develop these outflows is $\sim 1$ Myr (Sakamoto et al., 2014).

Numerical simulations have begun to study the radiative mode, depending however on small-scale recipes, which are calibrated on observations. Recipes are required to take into account the black hole growth and its associated feedback, all being sub-grid physics. Different groups do not converge to the same conclusions. While Springel et al. (2005) and Hopkins et al. (2006) found a good coupling between the AGN and the galaxy, Gabor and Bournaud (2014) conclude that the quasar mode has no quenching effect. Observations are therefore key to solve the issue of the AGN quenching efficiency.

\section{COOL-CORE CLUSTERS}

One example where AGN feedback is clearly demonstrated is found in the center of cool-core clusters. It has been known for a long time that the cooling time-scale of the hot ICM gas becomes smaller than the Hubble time in the center, and cooling flows are expected. However, only $10 \%$ of the expected cooling rate is observed, and this is now understood to be due to the radio jets of the central AGN reheating the gas. The jets carve cavities in the ICM, and uplift some hot gas. The denser regions around cavities cool in filaments, which infall after losing their pressure support, and are conspicuous in $\mathrm{H} \alpha$ (shocks) and molecular gas (Salomé et al., 2006, 2008).

Although most $\mathrm{H} \alpha$ is excited by shocks, there are some clumps of star formation (Canning et al., 2014). The observed scenario is far from the simple model of cooling flows, where most of the cooling occurred in the center. In real clusters, gas cools in the border of cavities, which can occur 20-50 kpc from the center. When the central galaxies is not at rest but oscillating in the cluster potential well, a cooling wake extends over $\sim 50 \mathrm{kpc}$ (Salomé P and Combes, 2004; Russell et al., in press). In this
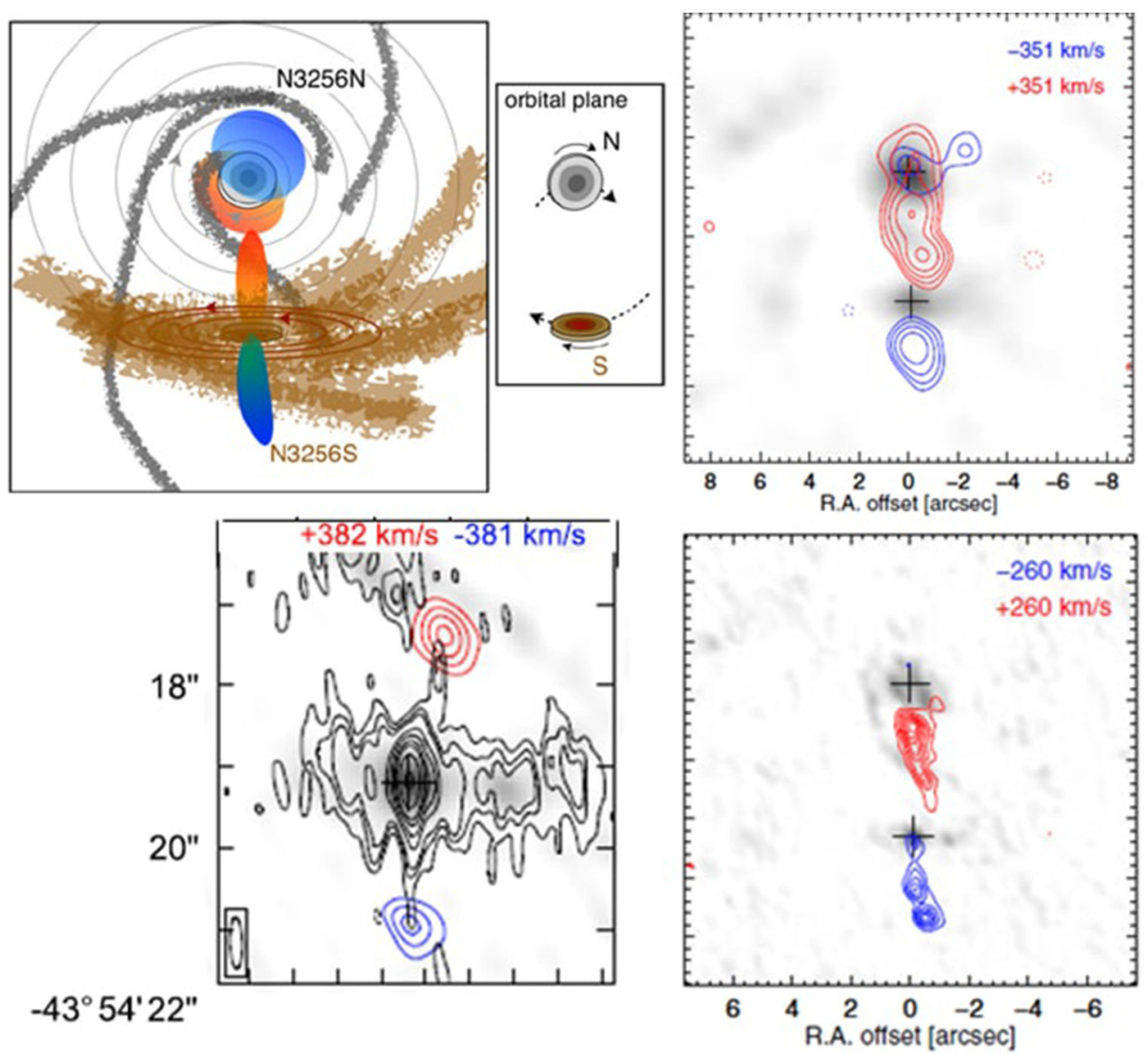

FIGURE 1 | ALMA observations of two molecular outflows in the merging galaxies NGC3256 (sketched at top left), adapted from Sakamoto et al. (2014), figure reproduced with permission. The northern object, almost face-on, reveals an outflow toward the observer, while the southern galaxy, nearly edge-on, shows a collimated outflow in the plane of the sky. The latter appears to coincide with a small radio jet, according to the VLA $3.6 \mathrm{~cm}$ radio map at the bottom left. The two CO maps at the right (top at low resolution, and bottom at high resolution) show that the northern outflow is resolved out. It must be a wide-angle outflow, uncollimated. 
complex picture, gas inflow and outflow coexist; the molecular gas coming from previous cooling is dragged out by the AGN feedback, and can explain the large metallicity, necessary to detect the CO lines. The uplifted bubbles of hot gas create inhomogeneities and further cooling, and the cooled gas fuels the AGN, to close the loop. Through ram-pressure forces, the cold gas velocity is much lower than free-fall (Salomé et al., 2008).

\section{MOLECULAR OUTFLOWS}

Molecular outflows are now frequently observed in nearby galaxies, and statistics have been made with respect to their starburst or AGN origin (Cicone et al., 2014). For AGN-host galaxies, the outflow rate correlates with the AGN power, and also the $\mathrm{L}_{A G N} / \mathrm{L}_{b o l}$ luminosity ratio ( $\mathrm{L}_{b o l}$ being the total luminosity of the galaxy, including the AGN). The correlation does not exist for starbursts. What is also highly interesting is the good correlation between the momentum carried in the outflow (vdM/dt, where $\mathrm{v}$ is the outflow velocity )with the photon momentum output of the $\mathrm{AGN} \mathrm{L}_{A G N} / \mathrm{c}$. The average value is $\mathrm{vdM} / \mathrm{dt} \sim 20 \mathrm{~L}_{A G N} / \mathrm{c}$, which is only possible with energy-driven outflows (Zubovas and King, 2012), i.e., when the energy injected by the inner wind is fully conserved throughout the outflow, unless the outflowing shell is optically thick to the infrared radiation, implying high momentum flux (Ishibashi and Fabian, 2015).

For the radio mode to be efficient in quenching star formation, there must be a strong coupling with galaxy disks. This is the case when the radio jet is not perpendicular to the galaxy plane, but is inclined so that the jet can sweep out some significant region in the disk. For example, the radio jet starts its way in the plane of the Seyfert 2 galaxy NGC1068: a molecular outflow of 63 $\mathrm{M}_{\odot} / \mathrm{yr}$, about 10 times the SFR has been observed by ALMA in the circum nuclear disk region (Garcia-Burillo et al., 2014). In the extreme case of IC5063, the jet is entirely in the plane, and creates secondary outflow features, at each collision with in-plane clouds (Morganti et al., 2015; Dasyra et al., 2016). Some of the gas might be optically thin in theses flows.

Feedback is also observed in low-luminosity AGN. One of the smallest outflow detected up to now is that of the Seyfert 2 NGC1433, with a maximum outflow of $100 \mathrm{~km} / \mathrm{s}$, along the minor axis (Combes et al., 2013). The case of the lenticular NGC1377 is very puzzling (Aalto et al., 2016). There is no radio AGN emission, although a very collimated molecular outflow is detected, with even a precession, visible since the outflow is almost in the plane of the sky (see Figure 2).

\section{JET-INDUCED STAR FORMATION}

The AGN feedback is frequently negative, but can be also positive, and trigger star formation. One of the most convincing examples of jet-induced star formation has been found in the Minkowski object (NGC541, distance of $82 \mathrm{Mpc}$ ), where conspicuous HII regions are observed at the extremity of the radio jet, outside of the optical galaxy (Croft et al., 2006). Molecular gas has been found in NGC541, but only an upper limit in the HII region with IRAM (Salomé et al., 2015), suggesting a high efficiency of star formation. Recently, $\mathrm{CO}$ emission was detected with ALMA (Lacy et al., 2017), compatible with the IRAM upper limit. However, when taken into account the low metallicity of the gas, which increases the $\mathrm{CO}$-to- $\mathrm{H}_{2}$ conversion ratio and thus the gas mass, and the excitation by shocks (reducing the SFR, for a given $\mathrm{H} \alpha$ flux), the star formation efficiency is now much lower in the triggered region.

A more nearby example of jet-induced star formation has been studied in Centaurus A, at a distance of $3.4 \mathrm{Mpc}$. Atomic gas has been mapped in shells around the galaxy by Schiminovich et al. (1994), and molecular gas has been found in those shells on the path of the radio jet by Charmandaris et al. (2000). Near the

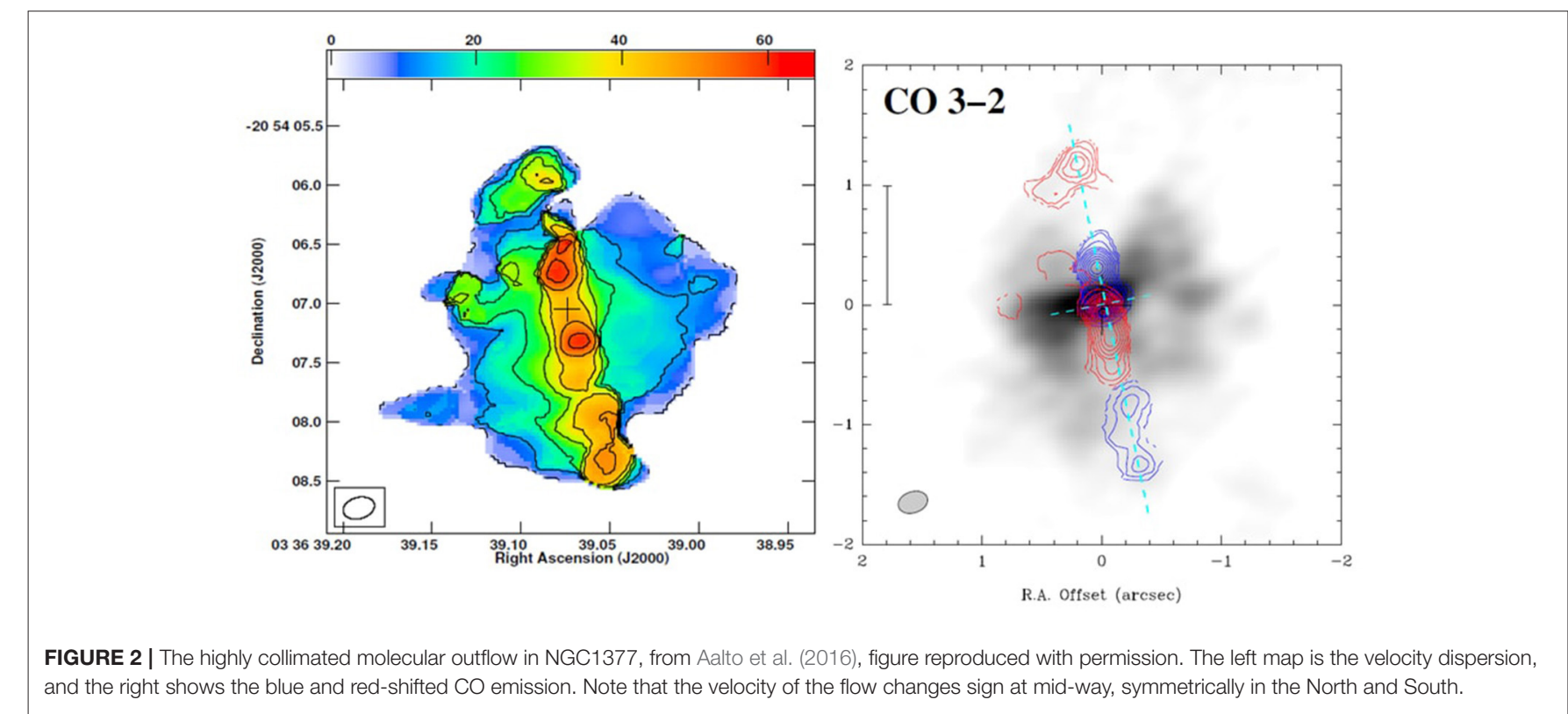



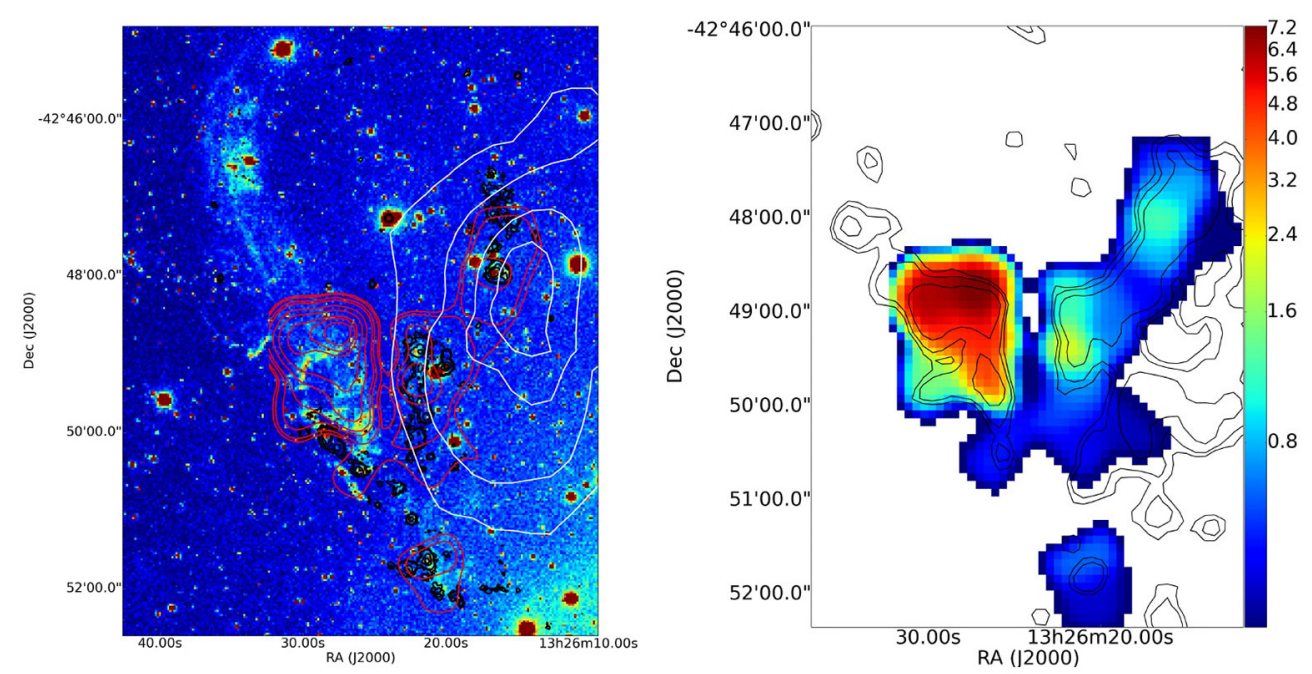

FIGURE 3 | Multi-phase filament in the radio jet of Centaurus A, from Salomé et al. (2016b), figure reproduced with permission. (Left) Superposed on the H $\alpha$ map, the red contours correspond to $\mathrm{CO}$ emission, the white to $\mathrm{HI}$, and the black to FUV from GALEX. (Right) The black HI contours are superposed on the CO emission map, showing that most of the molecular gas is outside the HI region.

northern shell, there is a conspicuous filament of star formation, aligned along the jet, mapped in $\mathrm{H} \alpha$, FUV-GALEX, and dust emission with Herschel. Molecular gas has been mapped with APEX and ALMA, and surprisingly, there is even more CO detected outside the HI shell that inside (Salomé et al., 2016b). The molecular gas is concentrated at the region where the jet encounters the HI shell, and the atomic gas is then transformed in the molecular phase under the impact of the jet pressure (see Figure 3). Ionized gas excitation was determined with MUSE spectral observations, with the help of the BPT diagnostics (Salomé et al., 2016a), and is mainly due to shocks, with some contribution of star formation.

There is clearly star formation triggering from the radio jet, however, the star formation efficiency is lower than in galaxy disks. This is a situation comparable to what is found in the outer parts of galaxies, where gas layers are flaring (e.g., DessaugesZavadsky et al., 2014). The reason might be a lack of pressure, due to low or absent restoring force from a stellar disk. The importance of pressure, and of the surface density of stars for the star formation efficiency has been emphasized by Blitz and Rosolowsky (2006) and Shi et al. (2011).

\section{CONCLUSIONS}

AGN feedback is required to quench star formation in massive galaxies, to reproduce the observed galaxy mass function, and avoid the over-production of very massive galaxies in cosmological simulations. One can consider two types of

\section{REFERENCES}

Aalto, S., Costagliola, F., Muller, S., Sakamoto, K., Gallagher, J. S., Dasyra, K., et al. (2016). A precessing molecular jet signaling an obscured,
AGN feedback: the quasar mode more frequent at high redshift, and the radio mode, more easy to observe in nearby galaxies.

One environment where the AGN feedback efficiency is clear is represented by cool core clusters, where the radio jets of the central bright galaxy carve bubbles and cavities in the hot intra-cluster gas, and moderate the gas cooling. Nearby galaxies frequently reveal significant molecular outflows, with a loading factor, the ratio between the outflow rate and the star formation rate, between 1 and 10 . Given the high momentum rate, the outflows appear to be energy conserving.

AGN feedback can also be positive. Some evidence of jetinduced star formation has been observed. In particular, the high jet pressure can trigger the phase transformation from atomic to molecular gas, favoring star formation. This triggered star formation is however less efficient than in normal galaxy disks.

\section{AUTHOR CONTRIBUTIONS}

The author confirms being the sole contributor of this work and approved it for publication.

\section{ACKNOWLEDGMENTS}

All appropriate permissions have been obtained from the copyright holders of the figures reproduced in the manuscript. Many thanks to Mauro d'Onofrio and the organizing committee for this exciting conference on Quasars in Padova, on April 2017. 
Canning, R. E. A., Ryon, J. E., Gallagher, J. S., Kotulla, R., O’Connell, R. W., Fabian, A. C., et al. (2014). Filamentary star formation in NGC 1275. Month. Notices $R$. Astron. 444, 336-349. doi: 10.1093/mnras/stu1191

Charmandaris, V., Combes, F., and van der Hulst, J. M. (2000). First detection of molecular gas in the shells of CenA. Astron. Astrophys. 356, L1-L4.

Cicone, C., Maiolino, R., Sturm, E., Graci-Carpio, J., Feruglio, C., Neriet, R., et al. (2014). Massive molecular outflows and evidence for AGN feedback from CO observations. Astron. Astrophys. 562:25. doi: 10.1051/0004-6361/201322464

Combes, F., Garcia-Burillo, S., Casasola, V., Hunt, L. K., Krips, M., Baker, A. J., et al. (2013). ALMA observations of feeding and feedback in nearby Seyfert galaxies: an AGN-driven outflow in NGC 1433. Astron. Astrophys. 558:11. doi: 10.1051/0004-6361/201322288

Croft, S., van Breugel, W., de Vries, W., Dopita, M., Martin, C., Morganti, R., et al. (2006). Minkowski's object: a starburst triggered by a radio jet. Astrophys. J. 647, 1040-1055. doi: 10.1086/505526

Dasyra, K. M., Combes, F., Oosterloo, T., Oonk, J. B. R., Morganti, R., Salomé, P., et al. (2016). ALMA reveals optically thin, highly excited CO gas in the jet-driven winds of the galaxy IC 5063. Astron. Astrophys. 595:4. doi: 10.1051/0004-6361/201629689

Dessauges-Zavadsky, M., Verdugo, C., Combes, F., and Pfenniger, D. (2014). CO map and steep Kennicutt-Schmidt relation in the extended UV disk of M 63 . Astron. Astrophys. 566:12. doi: 10.1051/0004-6361/201323330

Fabian, A. C. (2012). Observational evidence of active galactic nuclei feedback. Ann. Rev. Astron. Astrophys. 50, 455-489. doi: 10.1146/annurevastro-v081811-125521

Gabor, J. M., and Bournaud, F. (2014). Active galactic nuclei-driven outflows without immediate quenching in simulations of high-redshift disc galaxies. Month. Notices R. Astron. 441, 1615-1627. doi: 10.1093/mnras/stu677

Garcia-Burillo, S., Combes, F., and Usero, A. (2014). Molecular line emission in NGC 1068 imaged with ALMA. I. An AGN-driven outflow in the dense molecular gas. Astron. Astrophys. 567:24. doi: 10.1051/0004-6361/201423843

Hopkins, P., Hernquist, L., Cox, T. J., Di Matteo, T., Robertson, B., Springel, V., et al. (2006). A Unified, merger-driven model of the origin of starbursts, quasars, the cosmic X-ray background, supermassive black holes, and galaxy spheroids. Astrophys. J. Suppl. 163, 1-49. doi: 10.1086/499298

Ishibashi, W., and Fabian, A. C. (2015). AGN feedback: galactic-scale outflows driven by radiation pressure on dust. Month. Notices R. Astron. 451, 93-102. doi: $10.1093 / \mathrm{mnras} / \mathrm{stv} 944$

Lacy, M., Croft, S., Fragile, C., Wood, S., and Nyland, K. (2017). ALMA observations of the interaction of a radio jet with molecular gas in Minkowski's object. Astrophys. J. 838:9. doi: 10.3847/1538-4357/aa65d7

Morganti, R., Oosterloo, T., Oonk, J. B. R., Frieswijk, W., and Tadhunter, C. (2015). The fast molecular outflow in the Seyfert galaxy IC 5063 as seen by ALMA. Astron. Astrophys. 580:11. doi: 10.1051/0004-6361/201525860
Russell, H. R., McNamara, B. R., Fabian, A. C., Nulsen, P. E. J., Combes, F., Edge, A. C., et al. (in press). Close entrainment of massive molecular gas flows by radio bubbles in the central galaxy of Abell 1795. Month. Notices R. Astron.

Sakamoto, K., Aalto, S., Combes, F., Evans, A., and Peck, A. (2014). An infraredluminous merger with two bipolar molecular outflows: ALMA and SMA observations of NGC 3256. Astrophys. J. 797:28.

Salomé P., and Combes F. (2004). Mapping the cold molecular gas in a cooling flow cluster: Abell 1795. Astron. Astrophys. 415, L1-L5.

Salomé, P., Combes, F., Edge, A. C., Crawford, C., Erlund, A., Fabian, A. C., et al. (2006). Cold molecular gas in the Perseus cluster core. Association with Xray cavity, H? filaments and cooling flow. Astron. Astrophys. 454, 437-445. doi: 10.1051/0004-6361:20054745

Salomé, P., Combes, F., Revaz, Y., Edge, A. C., Hatch, N. A., Fabian, A. C., et al. (2008). Cold gas in the Perseus cluster core: excitation of molecular gas in filaments. Astron. Astrophys. 484, 317-325. doi: 10.1051/0004-6361:200809493

Salomé, Q., Salomé, P., and Combes, F. (2015). Jet-induced star formation in 3C 285 and Minkowski's Object. Astron. Astrophys. 574:9. doi: 10.1051/0004-6361/201424932

Salomé, Q., Salomé, P., Combes, F., Hamer, S., and Heywood, I. (2016a). Star formation efficiency along the radio jet in Centaurus A. Astron. Astrophys. 593:13. doi: 10.1051/0004-6361/201526409

Salomé, Q., Salomé, P., Combes, F., and Hamer, S. (2016b). Atomic-to-molecular gas phase transition triggered by the radio jet in Centaurus A. Astron. Astrophys. 595:14. doi: 10.1051/0004-6361/201628970

Schiminovich, D., van Gorkom, J., van der Hulst, T., and Kasow, S. (1994). Discovery of neutral hydrogen associated with the diffuse shells of NGC 5128 (centaurus A). Astrophys. J. 423, L101-L104. doi: 10.1086/187246

Shi, Y., Helou, G., Yan, L., Armus, L., Wu, Y., Papovich, S., et al. (2011). Extended schmidt law: role of existing stars in current star formation. Astrophys. J. 733:15.

Springel, V., Di Matteo, T., and Hernquist, L. (2005). Modelling feedback from stars and black holes in galaxy merger. Month. Notices R. Astron. 361, 776-794.

Zubovas, K., and King, A. (2012). Clearing out a galaxy. Astrophys. J. 745:5. doi: 10.1111/j.1365-2966.2005.09238.x

Conflict of Interest Statement: The author declares that the research was conducted in the absence of any commercial or financial relationships that could be construed as a potential conflict of interest.

Copyright (C) 2017 Combes. This is an open-access article distributed under the terms of the Creative Commons Attribution License (CC BY). The use, distribution or reproduction in other forums is permitted, provided the original author(s) or licensor are credited and that the original publication in this journal is cited, in accordance with accepted academic practice. No use, distribution or reproduction is permitted which does not comply with these terms. 